At first it seems surprising to find competition between two such dissimilar ions as an alkaline earth cation, calcium, and an alkali eation, lithium. However, it is recognized that the small size and relatively large ionizing potential of lithium cause some resemblance between it and the alkaline earth cations $^{6}$. An instance illustrating this resemblance is the low solubility of the carbonate and phosphate of lithium. Another example concerns the expansion of mica type crystal lattices as it depends on the interlayer cations. Barshad ${ }^{7}$ has shown that montmorillonite saturated with lithium is similar in swelling behaviour and differential thermal analysis to samples with alkaline earth interlayer cations, and differs characteristically from samples saturated with the other alkali cations or ammonium ions. Finally, lithium, like calcium, increases the rate of rubidium absorption by barley roots ${ }^{1}$.

These similarities seem to reflect ionic properties important in determining the 'fit' of the ions in molecular groupings of ligands. Lithium ions resemble the alkaline earth cations more than the other alkali cations in some of the properties that determine chemical affinities, including affinity for the carrier sites involved in active ion transport.

\section{Emanuel Epstein}

\section{Department of Soils and Plant Nutrition,} University of California, Davis, California.

${ }^{1}$ Epstein, E., and Hagen, C. E., Plant Physiol., 27, 457 (1952).

2 Epstein, E., and Leggett, J. E., Amer. J. Bot., 41, 785 (1954).

s Jacobson, L., Moore, D. P., and Hannapel, R. J., Plant Physiol. (in the press).

"Overstreet, R., Jacobson, L., and Handley, R., Plant Physiol., 27, $583(1952)$.

${ }^{5}$ Sutcliffe, J. F., Biol. Rev., 34, 159 (1959).

${ }^{6}$ Latimer, W. M., and Hildebrand, J. H., "Reference Book of Inorganic Chemistry", third ed. (The Macmillan Co., New York, 1951).

'Barshad, I., Amer. Mineralogist, 35, 225 (1950),

\section{RADIOBIOLOGY}

\section{Resistance of Algae to Radiation}

EXPERIMENTs carried out since 1954 have shown that various algae are much more resistant to radiation than are higher plants and animals. The results, which will be published fully elsewhere, are as follows :

$\quad$ Algae
Spirogyra
Zygnema, Mougeotia
Cosmarium
Euglena
Eudorina
Chlorella
Chaetomorpha
Prorocentrum

Author
Godward, 1954, and unpub.
Prasad, 1959, unpub.
Nizam, 1959, unpub.
Leedale, 1958
Rayns, 1959, unpub.
Nizam, 1959, unpub.
Patel, 1959, unpub.
Dodge, 1959, unpub.

Dose survived
(rads)
20,000 plus
$50,000-70,000$
70,000
150,000
100,000
wo successive doses
of $1,000,000$
50,000 plus
2,000

Of the above, only the last-mentioned, Prorocentrum, has the same order of sensitivity as the higher plants, if one excepts their specially resistant parts such as pollen grains and seeds. In all the experiments the algae were maintained in culture as long as possible after radiation; the recovery could follow in a matter of days but might need up to five weeks. Special features of the nuclear cytology probably account for the resistance and rapid recovery of the Conjugales $2,3,5$ and Euglena ${ }^{4}$. These features do not occur in Eudorina, which has, however, very small chromosomes (ref. 1 and Rayns, unpublished work). Smaller still and unresolvable with the visual light microscope except at prophase, and even then uncountable, are those of Chlorella, which it seems impossible to kill. On the other hand, the chromosomes of Prorocentrum are relatively large, and it would seem that, like those of the higher plants in general, they may have a localized centromere. In those algae where the chromosome organization is conventional or at any rate gross, it seems that resistance to radiation is relatively low. No organism has, to my knowledge, been reported as surviving an experimental dose such as that given above to Chlorella, where the recovery process was, however, long; although it seems clear that the marine algae which received considerable radiation from atomic explosions were not killed.

The radiations were carried out at the Medical Research Council Unit, Hammersmith Hospital.

Department of Botany,

Queen Mary College,

Mile End Road,

London, E.1.

${ }^{1}$ Cave, M., and Pocock, M. A., Amer. J. Bot., 38, 800 (1951).

${ }^{2}$ Godward, M. B, E., Proc. Int. Bot. Congr., Paris, 1954 ; Heredity. 11 (1957).

${ }^{s}$ King, G. C., New Phyt. (in the press).

Leedale, G. F., Archiv Mikrobiol., 32, 32 (1958).

${ }^{5}$ Prasad, B. N., thesis, Ph.D., London (1959).

\section{Radiocarbon Dating of Soil Humus}

THE turn-over of soil organic matter is a much. discussed problem in pedology. Although much has been written about the rate of breakdown of soil organic matter in mull and mor (for references see ref. 1), our knowledge of the turn-over of forest soil humus is very incomplete. This is particularly true of the organic matter in soil horizons below the humus layer.

The radiocarbon dating method opens a way to determine the approximate 'time of residence' in the soil of the organic matter, supposing that this time is of the order some hundred years or more. For this reason three soil samples from a profile in Garpenberg, Province of Dalarna, Middle Sweden, have been studied. The sample plot was a goodgrowing 95-year old spruce forest on moraine, altitude $200 \mathrm{~m}$. (just above the so-called highest coastline). The samples represented the mor layer $\left(A_{0}\right)$, the leached but humus-coloured top horizon of the mineral soil $\left(A_{1}\right)$, and the brown accumulation horizon $(B)$. The profile, which will be described in more detail in a later publication, is a favourable type of the podzol profile; similar profiles are not uneommon on moraine soils in this region.

The samples were passed through a $3-\mathrm{mm}$. sieve leaving stones, gravel and all but the finest roots on the sieve. The sifting process passed 63 per cent of the $A_{0}, 90$ per cent of the $A_{1}$ and 76 per cent of the $B$ layers, based on samples collected in the field (measured dry).

The loss on ignition was 45.5 per cent of the $A_{0}$ sample, 15.9 of the $A_{1}$ and $5 \cdot 2$ of the $B$ sample. In $A_{0}$ and $A_{1}$ the loss on ignition can be considered as estimates of the organic matter content, which, however, is lower than $5 \cdot 2$ in the $B$ horizon.

The residual carbon-14 activity was measured with the following results : 